\title{
Questions and Answers and Guidelines in EBD Volume 5
}

\author{
Derek Richards \\ Editor, Evidence-based Dentistry
}

In the previous years, we have outlined the questions and answers addressed by summaries in $\mathrm{EBD}^{1,2}$. Here we list the two guidelines, 39 questions, answers and evidence levels of areas addressed in EBD volume five. Evidence levels ${ }^{3}$ are only given for those papers achieving level $3 \mathrm{~A}$ and above.

Evidence-Based Dentistry (2004) 5, 110-112. doi:10.1038/sj.ebd.6400303

\section{Guidelines}

How to ensure pain relief for patients with apical periodontitis?

How to ensure pain relief for patients with acute apical abscess?

Question
Caries
Are topical fluoride treatments (toothpastes, mouthrinses,
gels or varnishes) effective in reducing dental caries in
children and adolescence?
For the prevention of caries in children is there a beneficial
effect of adding topical fluoride therapy (TFT) in the form of
mouthrinse, gel or varnish to fluoride toothpaste?
Does the use of a pacifier increase the risk of early childhood
caries?

What are the trends in dental caries prevalence and severity in Latin America and the Caribbean?

How effective are fissure sealants in preventing occlusal caries in children and adolescents?

Are pit and fissure sealants effective in preventing decay in children and adolescents who are at risk of caries?

Is fluoride gel effective at preventing caries in low caries-risk children?

\section{Dental Public Health}

Are dental Health educators in general practice clinically and cost effective in improving oral health?

Is there an association between water fluoridation and Down's syndrome?

Does the use of bitewing radiographs provide additional benefit over clinical examination alone when assessing caries prevalence in populations of young people? Evidence Page level

Children and adolescents benefit from topical fluorides irrespective of water fluoridation or other sources of fluoride exposure.

Topical fluorides (mouthrinses, gels, or varnishes) used in addition to fluoride toothpaste achieve a modest reduction in caries compared to toothpaste used alone.

There is a clear need to improve the quality of research in order to discover any association between pacifier use and childhood caries.

This study showed some statistically significant downward trends in dental caries between 1970 and 2000 in 16 countries in Latin America and the Caribbean.

Resin-based sealants have a caries-preventive effect but more high-quality clinical trials are needed.

Sealants based on resins are effective for prevention of caries on occlusal surfaces of permanent molars. There is insufficient evidence to support the long-term routine use of sealants based on Gl.

Although this study provides evidence of a significant beneficial effect of fluoride gel treatment in the permanent teeth of young children at low risk of caries, the clinical relevance is considered low based on the large NNTs obtained from the trial.

This study showed no benefit to dental health but there toothbrushing skills.

The practitioner should be aware of any possible adverse effects of fluoridation but there is no conclusive evidence of association between water fluoridation and Down's syndrome.

There is insufficient evidence at present to indicate the
$1 \mathrm{~A}$

$1 \mathrm{~A}$

2B

$1 \mathrm{~A}$

$1 \mathrm{~A}$

$1 \mathrm{~B}$ the diagnostic yield from using bitewing radiographs in an epidemiological study of specific populations. 


\section{Endodontics}

In patients requiring apicectomy and retrograde obturation, which filing material is most effective?

\section{Implants}

What complications are associated with dental implants?

\section{Methodology}

What is the quality of systematic reviews in dentistry?

\section{Oral Cancer}

Are screening programmes effective in reducing mortality rates from oral cancer?

Are there any health risks associated with smokeless tobacco?

\section{Oral Medicine}

In people at increased risk of bacterial endocarditis does prophylactic penicillin before invasive dental procedures affect mortality, serious illness or endocarditis incidence?

Are oral appliances effective in the treatment of sleep apnoea in adults?

\section{Oral Surgery}

Does hypotensive anaesthesia reduce blood loss in orthognathic surgery?

Does the presence of third molars increase the risk of fracture of the mandibular angle?

\section{Orthodontics}

For patients requiring orthodontic treatment are outreach clinics an effective way of delivering services?

Do postal reminders reduce non-attendance in patients invited to an orthodontic clinic?

Do functional appliances enhance mandibular growth in the treatment of skeletal Class II malocclusions?

Does traditional orthodontic treatment change the prevalence of temporomandibular disorder?

Is the Jones Jig an effective method of distalising upper first permanent molars?

Is there any difference between pre-coated and non-pre-coated brackets with respect to bond failure rate and clinical time to place brackets?
Based on the outcome of two RCTs glass ionomer is as effective as amalgam. Therefore, at present the evidence is not strong enough to recommend a move from amalgam

It was not possible to calculate an overall incidence of complications for implant prostheses because there were no multiple clinical studies that simultaneously evaluated all or most of the categories of complications.

Although the increase in availability of systematic reviews in dentistry is welcome, care needs to be taken to ensure that these reviews are well conducted and clinically relevant.

No evidence to support or refute the use of visual screening as a method of screening for oral cancer?

Chewing betel quid and tobacco is associated with a substantial risk of oral cancers in India. Most recent studies from the US and Scandinavia are not statistically significant, but moderate positive associations cannot be ruled out due to lack of power.

There is no evidence about whether penicillin prophylaxis is effective or ineffective against bacterial endocarditis in people at risk who are about to undergo an invasive dental procedure.

Oral appliances should be the first choice of treatment They are feasible alternatives for patients who cannot be managed with nasal continuous positive airway pressure.

This study provides evidence of reduced blood loss with hypotensive surgery but the case for routine use has not yet been made convincingly

The presence of a lower third molar increases the risk of an angle fracture of the mandible.

There do not appear to be any marked advantage or orthodontics in outreach clinics

Postal reminders can improve attendance but more research is outpatient attendance.

Functional appliances can correct significant Class II

malocclusions, but this study was unable to conclude whether functional appliances enhance mandibular growth.

Current evidence shows no definite link between orthodontics and TMD but more research is required.

It is suggested that there is no advantage in using the Jones Jig as a non-compliance appliance.

Neither the precoated nor the non-pre-coated brackets evaluated were clinically superior in terms of bond failure rate in the first six months of fixed appliance treatment. The use of precoated brackets did not result in a significant reduction upon clinical bonding time. 
What is the effect of fluoridated elastomers on the quantity of disclosed dental plaque surrounding an orthodontic bracket? What is the most effective method of preventing white spot lesions during orthodontic treatment?

In patients undergoing orthodontic treatment is fluoride effective in preventing white spot lesions?

Does treatment started before adolescence, when followed by a second phase of treatment in the early permanent dentition, provide superior results to single-phase treatment delayed until adolescence?

Is the Twin-block orthodontic appliance effective in the early treatment of the developing Class II Division 1 malocclusion?

Does the Herbst appliance have any effect on temporomandibular joint morphology?

What is the best method of stabilising tooth position after orthodontic treatment?

\section{Pain}

Is the third molar pain model different from other models and does it effect analgesic response?

\section{Periodontology}

Is local delivery of tetracycline an effective option in the treatment of chronic periodontitis?

Is periodontal disease associated with cardiovascular disease?

Is unsupervised use of a triclosan/copolymer dentifrice effective in slowing the progression of periodontal disease in a normal adult population?

\section{Restorative Dentistry}

When patients' anterior teeth are stained, is direct or indirect veneer restoration most effective?

In patients with endodontically treated premolars are full cast crowns more effective than direct composite restorations?

How long do IPS-Empress ceramic restorations last?

\section{Temporomandibular Dysfunction}

Is stabilisation splint therapy effective in reducing symptoms in people who have pain dysfunction syndrome?
Fluoridated elastomers do not affect the quantity of disclosed plaque but may help control enamel demineralisation. For orthodontic patients undergoing fixed appliance therapy, oral hygiene measures should include toothbrushing with fluoridated toothpaste, augmented by daily application of high fluoride concentration gel or chlorhexidine mouthwash. The efficacy of fluoride release from bonding materials or elastomers in reducing decalcification is unverified as yet.

Local fluoride delivery during orthodontic treatment reduces white spot lesions; however, more evidence is needed on the best delivery method.

There are currently very few indications to justify early treatment for Class II malocclusions.

- Early treatment of Class II malocclusions is effective, but not efficient.

- The difference in skeletal and dental morphology achieved in early treatment disappeared almost completely after comprehensive treatment with fixed appliance was completed.

Skeletal change is not clinically significant, but the twin-block appliance is effective in reducing the overjet and severity of malocclusion.

More research is required to better understand the effects of the Herbst appliance on TMJ morphology.

There are insufficient research data on which to base our clinical practice on retention at present.

Post-surgical pain in different areas of the body appears to be similar in terms of sensitivity to non-narcotic analgesics. Therefore, dentists can rely on summary tables produced using several models of acute surgical pain

Local delivery agents should be used as an adjunct and not an alternative to mechanical therapy in the treatment of periodontal diseases.

There is insufficient evidence to suggest that periodontal disease screening and periodontal treatment in people at risk for CVD would reduce the incidence of CVD.

In normal adult population, unsupervised use of a triclosan/ copolymer dentifrice tended to slow progression of periodontal disease.

Patients should be advised that there is no 'best' type of veneer.

Endodontically treated teeth with minimal loss coronal structure may be restored with adhesion techniques. IPS-Empress inlay, onlays and anterior crowns show good
1. Lawrence A, Richards D. Questions answered in EBD Volumes 1 and 2. EvidenceBased Dent 2002; 3:27-29.

2. Richards D. Questions and Answers in EBD Volumes 3 and 4. Evidence-Based Dent 2003; 4:94-97.
3. Richards D. Not all evidence is created equal - so what is good evidence. Evidence-Based Dent 2003; 4:17-18. 